
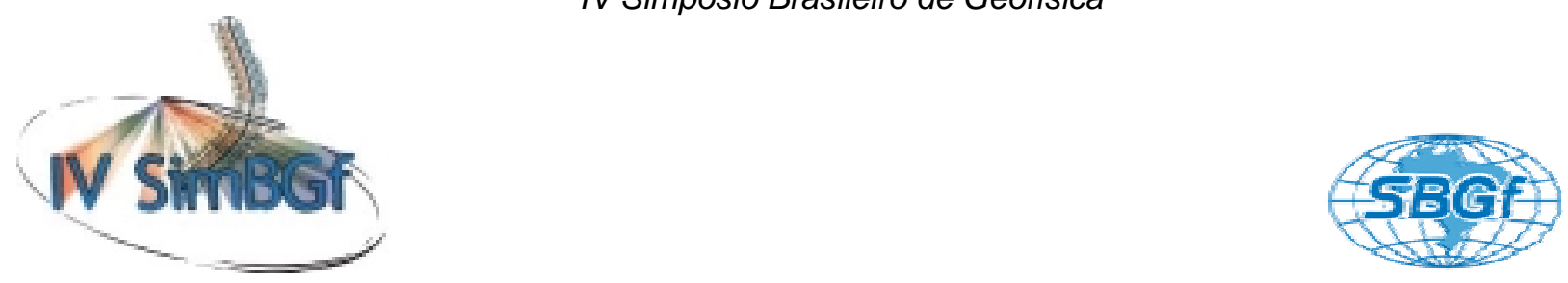

\title{
Interpretação gravimétrica do relevo do embasamento da Bacia de Taubaté através da inversão não linear com vínculo de suavidade
}

Raphael Di Carlo Silva dos Santos ${ }^{*}$, , Darcicléa Ferreira Santos ${ }^{(1)}$ e João Batista Corrêa da Silva ${ }^{(1)}$. 1- Universidade Federal do Pará (UFPA)

\section{Copyright 2010, SBGf - Sociedade Brasileira de Geofísica}

Este texto foi preparado para a apresentação no IV Simpósio Brasileiro de Geofísica, Brasília, 14 a 17 de novembro de 2010. Seu conteúdo foi revisado pelo Comitê Técnico do IV SimBGf, mas não necessariamente representa a opinião da SBGf ou de seus associados. É proibida a reprodução total ou parcial deste material para propósitos comerciais sem prévia autorização da SBGt.

\section{Abstract}

We present a gravity interpretation of the Taubaté basin basement relief. The residual Bouguer anomaly produced by the basin was estimated through a robust polynomial fitting method. We interpreted three gravity profiles across the basin strike using the smoothness constraint. We obtained average estimated basement depths between $370 \mathrm{~m}$ and $940 \mathrm{~m}$, indicating a very shallow basement.

\section{Introdução}

A Bacia de Taubaté localiza-se a leste do estado de São Paulo limitada pelas Serras do Mar e da Mantiqueira a sudoeste e a nordeste, respectivamente. Apresenta extensão de $170 \mathrm{~km}$ na direção SW-NE, largura de aproximadamente $25 \mathrm{~km}$ e profundidade média no entorno de 850 km, (Torres-Ribeiro e Borghi, 2005). A Bacia de Taubaté é uma bacia rift continental que compõe o sistema de Rifts da Serra do Mar.

Interpretações gravimétricas da Bacia de Taubaté têm sido realizadas para produzir informação sobre as interfaces crosta-manto e embasamento-sedimentos (Fernandes e Chang, 2001). Fernandes e Chang (2001) apresentam a interpretação gravimétrica do relevo do embasamento da Bacia de Taubaté obtendo: (1) o mapa residual de anomalia Bouguer usando os filtros de continuação para cima e passa-alta frequência; e (2) a modelagem gravimétrica a partir do cálculo do efeito gravimétrico de um corpo bidimensional de seção vertical arbitrária (Talwani et al., 1959), em perfis gravimétricos transversais à bacia.

Executamos neste trabalho a interpretação de três perfis gravimétricos transversais à Bacia de Taubaté. Neste trabalho usamos o método do ajuste robusto de polinômios (Beltrão et al., 1991) para obter a anomalia Bouguer residual devida à presença da bacia. A interpretação foi efetuada através da inversão não linear com o vínculo de suavidade nos perfis gravimétricos (Barbosa et al., 1997). A interpretação dos três perfis gravimétricos usando a metodologia de inversão não linear produz uma profundidade média do relevo do embasamento entre $370 \mathrm{~m}$ e $940 \mathrm{~m}$, evidenciando a presença de um embasamento raso.

\section{Localização dos Dados Gravimétricos}

O conjunto de dados gravimétricos da Bacia de Taubaté, utilizados na interpretação gravimétrica, foi retirada de uma cobertura de 1909 estações gravimétricas nas localizações aproximadas de $-48^{\circ} \mathrm{W},-44,5^{\circ} \mathrm{W},-24^{\circ} \mathrm{S}$ e $-22^{\circ} \mathrm{S}$. Os dados gravimétricos analisados da Bacia de Taubaté (Figura 1) localizam-se entre as coordenadas $-46,285^{\circ} \mathrm{W},-44,888^{\circ} \mathrm{W},-23,47^{\circ} \mathrm{S}$ e $-22,5^{\circ} \mathrm{S}$.

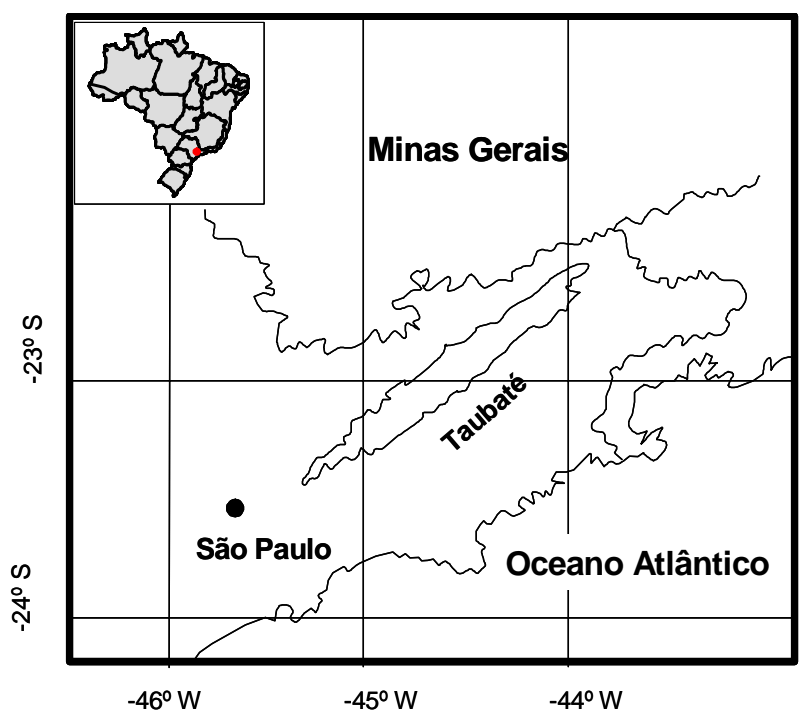

Figura 1 -Localização geográfica da Bacia de Taubaté

Para a obtenção da anomalia gravimétrica Bouguer completa foram utilizados o sistema de referência IGSN71, a Fórmula Internacional da Gravidade Geodetic Reference System - 1967 (Blakely,1995) e a densidade de $2,67 \mathrm{~g} / \mathrm{cm}^{3}$.

\section{Separação Regional-residual}

Para o cálculo da anomalia residual usamos o ajuste robusto de polinômios (Beltrão et al., 1991). Para determinar a ordem do polinômio que representa a componente regional devido às variações no relevo da interface crosta-manto empregamos os seguintes passos:

(1) Calculamos oito anomalias residuais, uma para cada ordem de polinômio, de um a oito. 
(2) Plotamos o erro médio quadrático (emq) do resíduo contra a ordem do correspondente polinômio (Figura 2).

(3) Adotamos como a ordem do polinômio, representando as variações da componente regional, aquela que produz, no gráfico obtido em (2), uma mudança abupta de gradiente. Adotamos, assim, de acordo com este critério, o polinômio de ordem quatro.

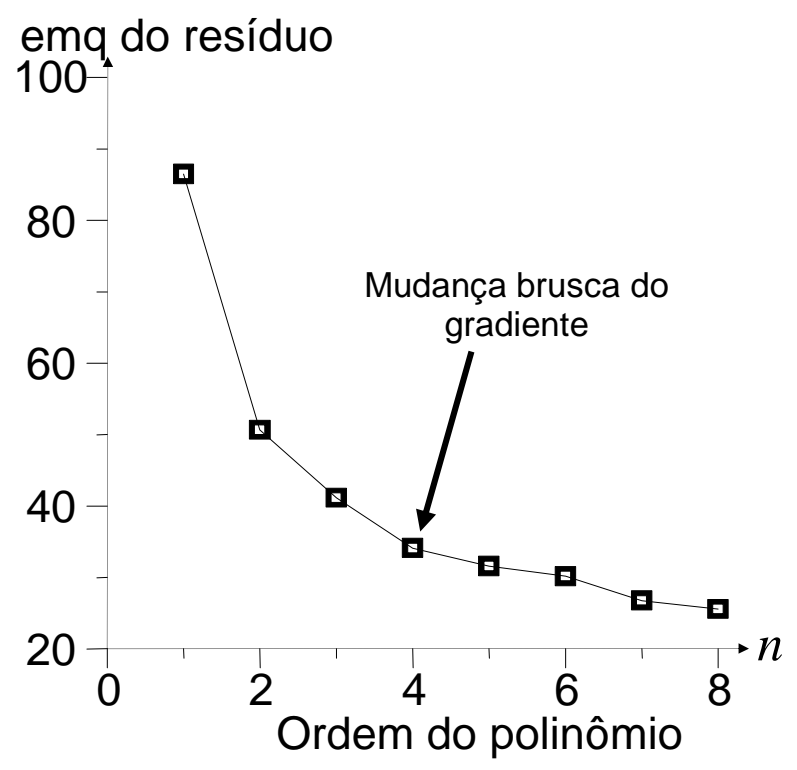

Figura 2 -Gráfico do emq do resíduo versus a ordem do polinômio.

Para retirar o possível efeito de fontes residuais intrassedimentares, aplicamos o método do ajuste robusto de polinômios (Beltrão et al.,1991) aos dados gravimétricos residuais. Para obter a ordem do polinômio, neste caso:

(1) Obtivemos as anomalias residuais associadas a polinômios ajustantes de ordens um até oito.

(2) Adotamos como critério de escolha da ordem do polinômio aquele cuja curva de contorno de $0 \mathrm{mGal}$ da correspondente anomalia residual está mais próxima dos limites horizontais da bacia. Adotamos assim, o polinômio de ordem seis, cuja anomalia residual é mostrada na Figura 3.

A anomalia Bouguer residual (Figura 3), mostra a presença de dois baixos gravimétricos na Bacia de Taubaté (Figura 3) sugerindo a presença de duas subbacias: uma localizada na porção NE e outra na região SW separadas por um alto estrutural (A, B e C, respectivamente, na Figura 3). Esta interpretação está de acordo com a descrição do arcabouço estrutural da Bacia de Taubaté descrito por Torres-Ribeiro e Borghi (2005).

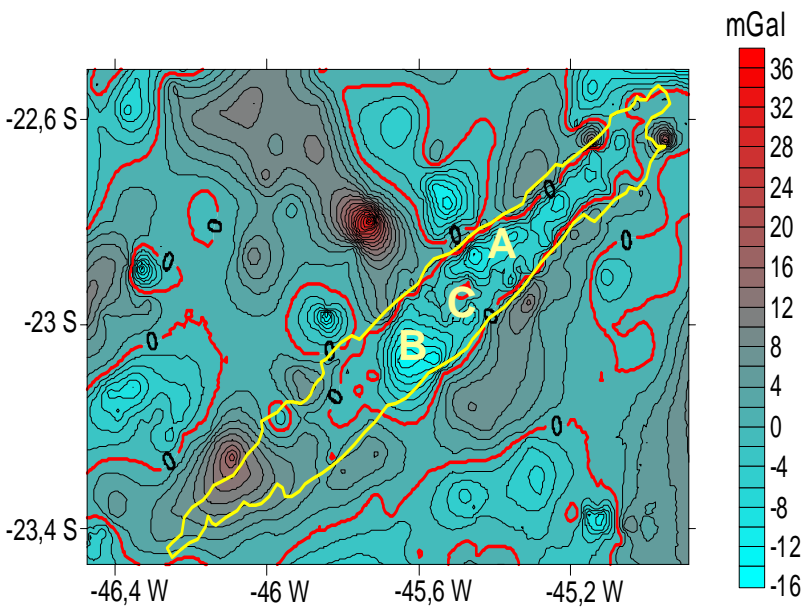

Figura 3 - Anomalia Bouguer residual com a localização da Bacia de Taubaté (curva amarela). Intervalo de contorno: $2 \mathrm{mGal}$.

\section{Inversão não linear da anomalia residual}

Para delinear a interface $S$ de separação entre os sedimentos e o embasamento (relevo do embasamento), Figura 4, a partir de um perfil de anomalia gravimétrica, vamos considerar:

(1) Que o contraste de densidade $\Delta \rho$ entre 0 embasamento e os sedimentos é constante e conhecido.

(2) Que o relevo do embasamento pode ser aproximado por um modelo interpretativo formado por um conjunto de $\mathrm{M}$ prismas bidimensionais verticais, justapostos, cujas espessuras representam as profundidades a ser estimada a partir da anomalia gravimétrica, Figura 4.

O problema de estimar as espessuras dos $M$ prismas (Figura 4) resulta em um problema não linear mal-posto no sentido de Hadamard (1902), uma vez que ele não apresenta solução estável.

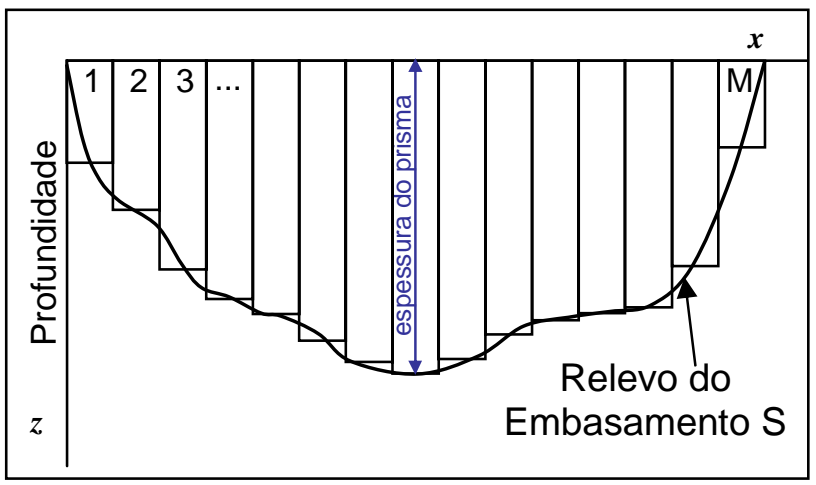

Figura 4 - Conjunto de M prismas verticais justapostos utilizado na interpretação da interface de separação $S$ entre o embasamento e os sedimentos. 
Para garantir a estabilidade da estimativa do relevo do embasamento introduzimos informação geológica, a priori, sobre a característica de suavidade do relevo do embasamento através do funcional estabilizador de Tikhonov de primeira ordem (Tikhonov e Arsenin, 1977), conhecido por suavidade global.

Este funcional estabelece que as estimativas das espessuras dos prismas, espacialmente contíguos, devem estar próximas entre si, introduzindo um grau de suavidade na estimativa do relevo do embasamento.

O problema não linear da inversão de dados gravimétricos para as espessuras dos prismas do modelo interpretativo é resolvido através do algoritmo de Marquardt (Marquardt, 1963).

O grau de suavidade imposto à estimativa do relevo do embasamento é controlado por um escalar $\mu$, chamado parâmetro de regularização. Quanto maior o valor de $\mu$, maior o grau de suavidade. O procedimento adotado para obter uma solução estável foi:

(1) Contaminar as observações gravimétricas com várias sequências distintas de ruído pseudo-aleatório;

(2) Adotar como melhor valor de $\mu$ o menor valor que produz estimativas estáveis do relevo. Uma solução é considerada estável quando as estimativas, obtidas para cada sequência de ruído e o mesmo valor fixo de $\mu$, estão próximas.

\section{Resultados da interpretação gravimétrica usando a técnica de inversão não linear}

Com o objetivo de interpretar a profundidade do relevo do embasamento suave na Bacia de Taubaté selecionamos três perfis cujas localizações são mostradas na Figura 5.

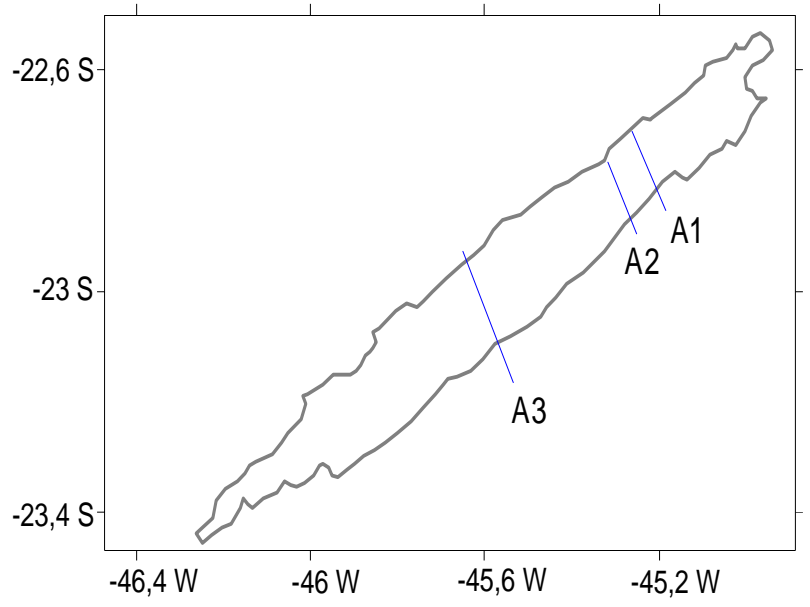

Figura 5 - Localização geográfica dos perfis $A 1, A 2$ e A3 e da Bacia de Taubaté.

As coordenadas geográficas dos extremos dos perfis são $\left(-45,262^{\circ} \mathrm{W},-22,711^{\circ} \mathrm{S}\right)$ e $\left(-45,183^{\circ} \mathrm{W},-22,855^{\circ} \mathrm{S}\right)$ para A1; $\left(-45,253^{\circ} \mathrm{W},-22,897^{\circ} \mathrm{S}\right)$ e $\left(-45,318^{\circ} \mathrm{W},-22,767^{\circ} \mathrm{S}\right)$ para A2 e; $\left(-45,652^{\circ} \quad W, \quad-22,929^{\circ}\right.$ S) e $(-45,536 \circ$ W, $-23,1660$ S) para A3. Estas coordenadas são usadas para selecionar os perfis gravimétricos A1, A2 e A3, Figura 6.

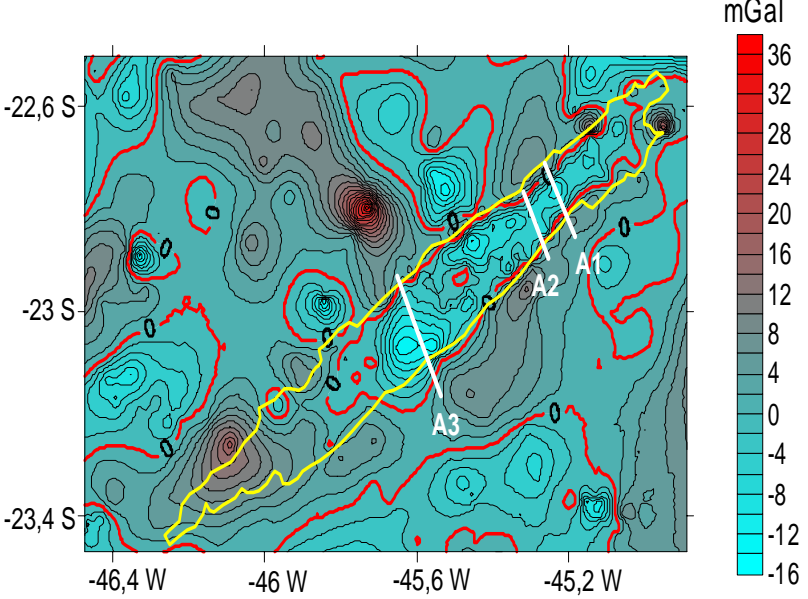

Figura 6 - Mapa Bouguer de anomalia residual apresentando a localização geográfica dos perfis gravimétricos A1, A2 e A3 e da Bacia de Taubaté.

Adotamos como contraste de densidade, entre o embasamento e 0 pacote sedimentar, 0 valor de $\Delta \rho=-0,34 \mathrm{~g} / \mathrm{cm}^{3}$ para a interpretação de todos os perfis gravimétricos mostrados na Figura 6 . Este valor de contraste de densidade $\Delta \rho$ foi definido por Fernandes e Chang (2001) como sendo o valor médio, calibrado para a porção central da Bacia de Taubaté a partir da seção sísmica e de um perfil gravimétrico.

Para aplicar o procedimento da inversão não linear nos perfis gravimétricos A1, A2 e A3 desprezamos as anomalias positivas localizadas nos extremos dos perfis.

Para verificar se as soluções são estáveis contaminamos as observações gravimétricas com ruído pseudo-aleatório Gaussiano com média nula e desvio padrão de 0,05 mGal. A interpretação dos perfis gravimétricos, no entanto, é feita na anomalia Bouguer residual sem a adição de ruído. Encontramos assim os seguintes valores para 0 parâmetro de regularização $\mu$ : 5 para os perfis gravimétricos A1 e A3 e 10 para o perfil gravimétrico A2.

Para todos os perfis gravimétricos tomamos um modelo interpretativo (Figura 4) com 100 prismas. A interpretação do relevo do embasamento é apresentada por uma linha contínua definindo uma poligonal. Os vértices desta poligonal são definidos pelas estimativas das profundidades dos prismas do modelo interpretativo (Figuras 7b, 8b e 9b).

A interpretação do perfil gravimétrico A1 (Figura 7a) mostra uma profundidade máxima de $747 \mathrm{~m}$ localizado na coordenada 12,4 km (Figura $7 \mathrm{~b}$ ). O perfil gravimétrico A2 (Figura 8a) apresenta profundidade média entre 370 $\mathrm{m}$ e $535 \mathrm{~m}$ (Figura 8b). O perfil gravimétrico A3 (Figura 9a) apresenta uma profundidade máxima de $940 \mathrm{~m}$ (Figura 9b).

\section{Conclusões}

Apresentamos neste trabalho a interpretação gravimétrica em três perfis localizados na Bacia de Taubaté.

IV Simpósio Brasileiro da SBGf - Brasília 2010 
A anomalia gravimétrica residual devida à presença da bacia foi obtida usando a metodologia do ajuste robusto de polinômios e permitiu identificar duas subbacias e um alto estrutural presentes na Bacia de Taubaté. Produzimos também estimativas estáveis do relevo do embasamento ao longo de três perfis transversais à bacia, utilizando a metodologia da inversão não linear com o vínculo de suavidade global. Os resultados apontam para profundidades médias do relevo do embasamento entre $370 \mathrm{~m}$ e $940 \mathrm{~m}$, evidenciando a presença de um embasamento raso. A interpretação gravimétrica apresentada acima neste trabalho foi consistente com análises estruturais da Bacia de Taubaté existentes na literatura.

Uma análise futura para a interpretação do relevo do embasamento da bacia de Taubaté deverá incluir a inversão de dados gravimétricos $3 D$ incorporando 0 vínculo da variação total, que permite obter estimativas de relevos descontínuos do embasamento. Este tipo de relevo é mais compatível com regime de forças distensivas geradoras desta Bacia.

\section{Agradecimentos}

Os autores agradecem ao Programa de Geofísica e Geologia Aplicado à Caracterização de Reservatório de Hidrocarbonetos (PRH-06) - Agência Nacional do Petróleo (ANP) e ao Conselho Nacional de Desenvolvimento Científico e Tecnológico (CNPq) pelo suporte financeiro em forma de bolsas de estudo e de pesquisa, respectivamente, e à Professora Naomi Ussami pela cessão do conjunto de dados gravimétricos da Bacia de Taubaté.

\section{Referências}

Barbosa, V. C. F.; Silva, J. B. C.; Medeiros, W. E. Gravity inversion of basement relief using approximate equality constraints on depths. Geophysics, v. 62, n.6, p. 17451757, 1997.

Beltrão, J. F.; Silva, J. B. C.; Costa, J. C. Robust polynomial fitting method for regional gravity estimation. Geophysics, v. 56, n. 1, p. 80-89, 1991.

Blakely, R. J. Potential theory in gravity and magnetic applications. Cambridge: Cambridge University Press, 1995. $441 \mathrm{p}$.

Fernandes, F. L. ; Chang, H. K. Modelagem gravimétrica da Bacia de Taubaté - Vale do Rio Paraíba do Sul, leste do estado de São Paulo. Brazilian Journal of Geophysics, v. 19 (2), p. 131-144, 2001.

Hadamard, J. Sur les problèmes aux dérivées partielles et leur signification physique. Bull Princeton Univ., V. 13, P. L-20, 1902.

Marquardt, D. W. An algorithm for least-squares estimation of nonlinear parameters. Journal Of The Society For Industrial And Applied Mathematics, V. 11, N. 2, P. 431-441, 1963.

Talwani, M.; Worzel, J. C.; Landisman, M. Rapid gravity calculations for two-dimensional bodies with application to the mendocino submarine fracture zone. J. Geophys. Res, v. 64, n. 1, p. 49-59, 1959.
Tikhonov, A. N.; Arsenin, V. Y. Solutions of ill-posed problems. Washington: V.H. Winston \& Sons, 1977. 258p.

Torres-Ribeiro, M.; Borghi, L. Bacias sedimentares Brasileiras: Bacia de Taubaté. Phoenix, n. 74, p. 1-6, 2005. 
(a)
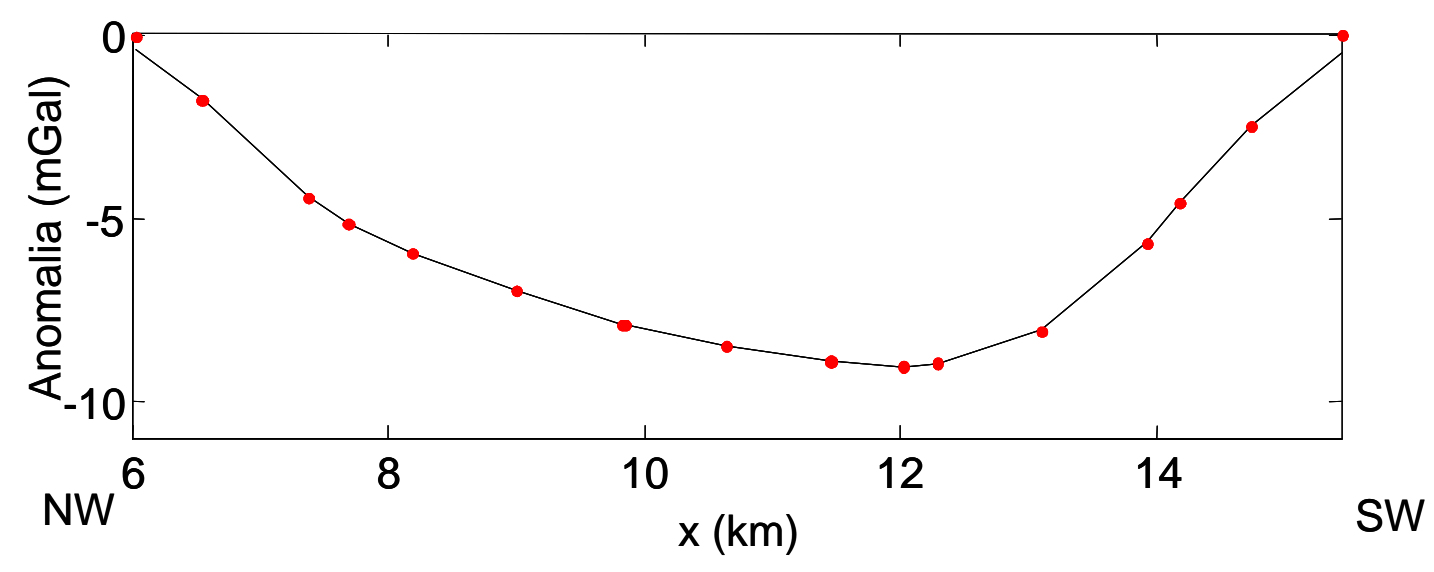

(b)

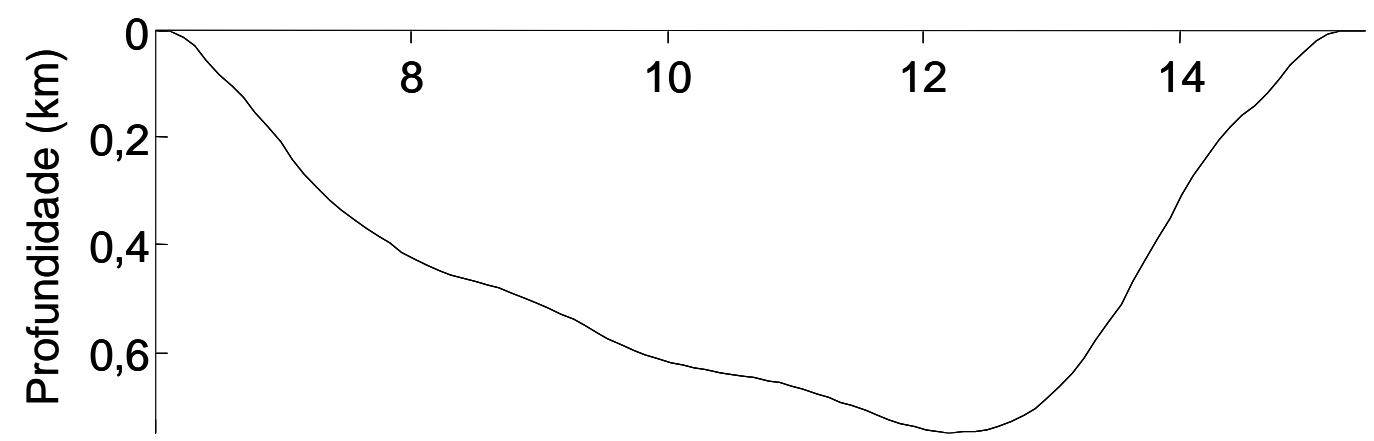

Figura 7 - Perfil gravimétrico A1 cuja localização é mostrada na Figura 5.: (a) anomalias gravimétrica observada (pontos vermelho) e ajustada (linha contínua preta). (b) Relevo estimado do embasamento.

(a)

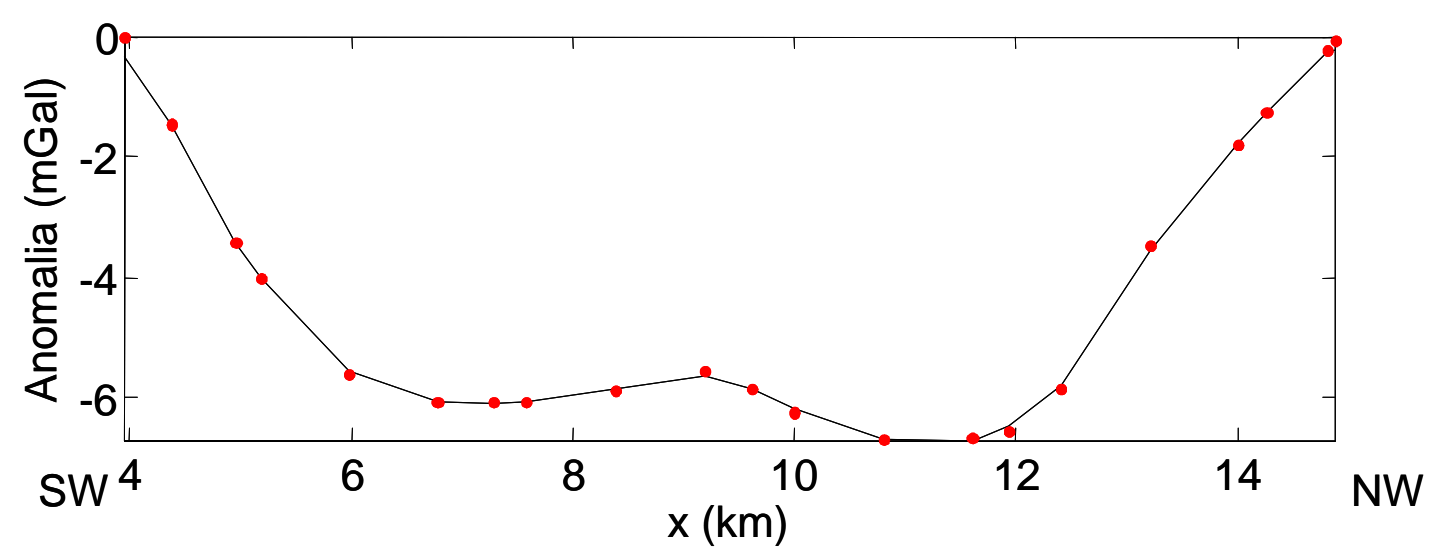

(b)

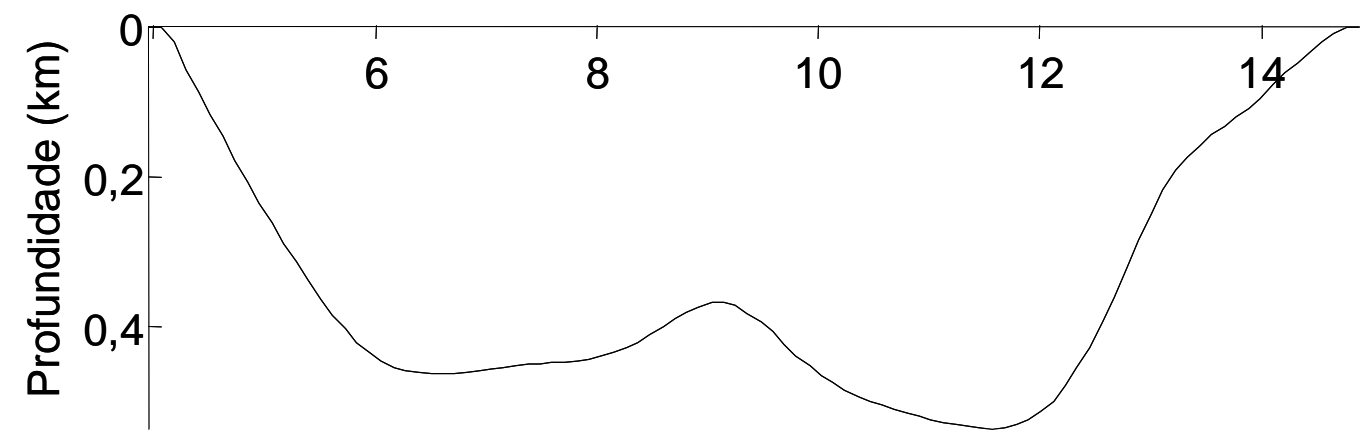

Figura 8 - Perfil gravimétrico A2 cuja localização é mostrada na Figura 5.: (a) anomalias gravimétrica observada (pontos vermelho) e ajustada (linha contínua preta). (b) Relevo estimado do embasamento. 


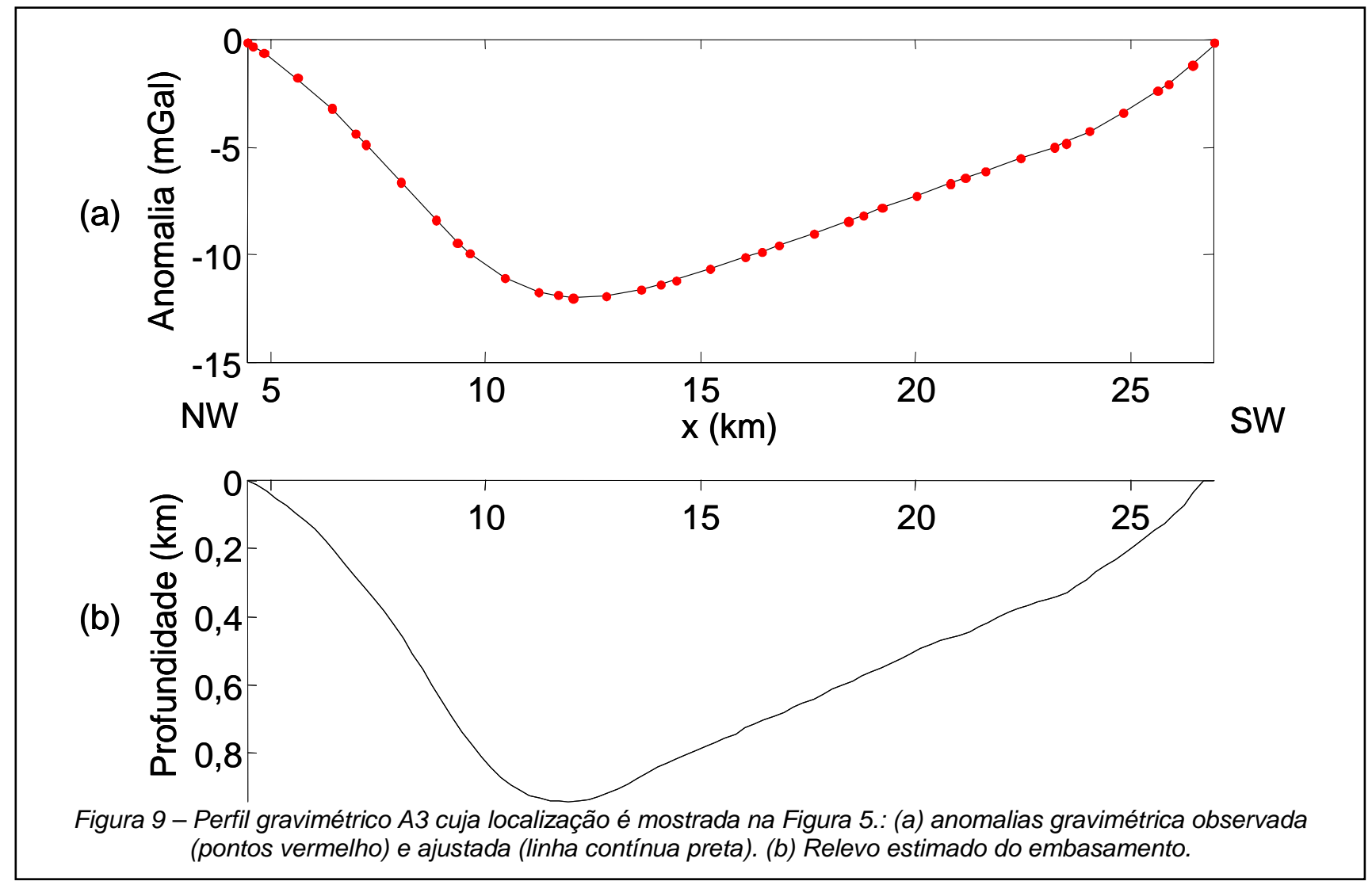

